\title{
MOFO PROVOCADO POR Aspergillus flavus E Penicillium spp., DURANTE A ARMAZENAGEM DE GRÃOS ÚMIDOS, EM DIFERENTES GENÓTIPOS DE MILHO
}

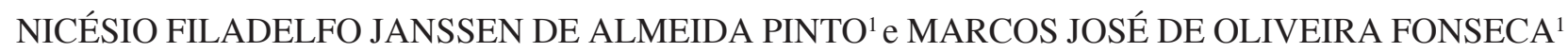 \\ ${ }^{1}$ Eng. Agr., Doutor, Pesquisador da Embrapa Milho e Sorgo, Núcleo de Manejo de Fatores Bióticos em Agroecossistemas \\ - NBIO. Rod. MG 424, km 65, C. Postal 151, 35701-970 Sete Lagoas, MG. nicesio@cnpms.embrapa.br
}

Revista Brasileira de Milho e Sorgo, v.5, n.3, p.450-455, 2006

\begin{abstract}
RESUMO - O objetivo deste trabalho foi avaliar o comportamento de grãos de diversos genótipos de milho, colhidos úmidos e armazenados em sistemas com e sem aeração, em relação ao mofo provocados por Aspergillus flavus e Penicillium spp. As seguintes cultivares/percentagens de umidade na colheita foram utilizadas: BR $206(17,2)$, BRS 2114 (18,3), BRS 2110 (18,5), BRS 1001 (19,1), BRS 2020 (19,2), BRS 3151 (19,5), BRS 3123 (19,5), BRS 1010 (21,2), BRS 3060 (21,3), BRS 3003 (21,4), CMS 200.122 $(21,9)$ e BRS $1030(22,9)$. Imediatamente após a colheita e a pré-limpeza, os grãos foram submetidos a armazenagem, em sistema com aeração (SCA) e sem aeração (SSA). O delineamento experimental foi o inteiramente casualizado, com 24 tratamentos, em três repetições. Determinou-se o teor de umidade e a porcentagem de grãos visualmente com mofo aos 2, 6 e 9 dias de armazenagem. A temperatura intergranular foi monitorada diariamente, variando, no SCA, de 21,2 a $22,8{ }^{\circ} \mathrm{C}$ e, no SSA, de 27,9 a $37,1{ }^{\circ} \mathrm{C}$. As cultivares BRS 1010 e BRS 3060, embora colhidas com a mesma umidade, diferiram significativamente entre si, com relação ao mofo dos grãos, aos 2, 6 e 9 dias de armazenagem, no SSA, e aos 6 e 9 dias, no SCA. Por outro lado, as cultivares BRS 3060 e BR 206, colhidas com umidades diferentes, não diferiram entre si, em ambos os sistemas e períodos de armazenagem, com relação ao mofo em seus grãos. Assim, durante a armazenagem verificou-se que, em relação ao mofo dos grãos provocado por A. flavus e Penicillium spp., além do conteúdo de umidade dos grãos, há diferenças significativas de comportamento entre os genótipos de milho.
\end{abstract}

Palavras-chave: Zea mays, fungos, mofo, resistência genética

\section{MOLD CAUSED BY Aspergillus flavus AND Penicillium spp. DURING STORAGE OF HUMID GRAINS IN DIFFERENT CORN GENOTYPES}

\begin{abstract}
The work had the objective of evaluating the behavior of several corn grain genotypes, in grains harvested while humid and stored in systems with continuous aeration and without aeration, in relation to the grain mold production for Aspergillus flavus and Penicillium spp. The following relationships between cultivars/moisture content were used: BR 206 (17.2\%), BRS 2114 (18.3), BRS 2110 (18.5), BRS 1001 (19.1), BRS 2020 (19.2), BRS 3151 (19.5), BRS 3123 (19.5), BRS 1010 (21.2), BRS 3060 (21.3), BRS 3003 (21.4), CM 200.122 (21.9) and BRS 1030 (22.9). Immediately after the harvest and grain pre-cleaning, these were submitted to the storage in continuous aeration system
\end{abstract}


- CAS - and in without aeration system - WAS. A completely randomized experimental design was used with 24 treatments in 3 replications. The moisture content and the percentage of grains visually moldy were determined 2, 6 and 9 days after the storage. The temperature in both systems were monitored daily, varying in CAS from 21.2 to $22.8{ }^{\circ} \mathrm{C}$; and in WAS from 27.9 to $37.1{ }^{\circ} \mathrm{C}$. The BRS 1010 and BRS 3060 cultivars harvested with the same moisture content, differed significantly amongst themselves in relation to the grains visually moldy at 2, 6 and 9 days WAS storage and 6 and 9 days in CAS storage. On the other hand, the BRS 3151 and BRS 3123 cultivars harvested with different humidity content did not differ amongst themselves, in both systems and storage periods in relation to the mold in their grains. In this manner, during the storage it was verified that in relation to the grain mold caused by A. flavus and Penicillium spp., in addition to moisture content of the grains, there are significant differences of behavior among the corn genotypes.

Key words: Zea mays, fungi, grain mold, genetic resistance

Em vista da inserção da cultura do milho em áreas de pivô central, do cultivo do milho "safrinha" e da intensificação na utilização de colhedoras automotrizes, há necessidade de informações sobre a qualidade e a segurança alimentar dos grãos, os quais têm sido colhidos com 25 a $30 \%$ de umidade, em base úmida. O tempo decorrido entre a colheita e a secagem pode ser suficiente para a colonização dos grãos pelos fungos de armazenamento.

De acordo com Alves et al. (2001), a qualidade dos grãos de milho da cultivar AG 1051, com relação às análises de índice de danos, índices de trincas e teste de suscetibilidade à quebra, foi reduzida à medida que se aumentou o teor de umidade de colheita. Os teores de umidade de colheita utilizados foram de 25, 22, 16,5 e 15\%, em base úmida, sendo os grãos secados até o teor de umidade final de $13 \%$, fumigados e armazenados em recipiente metálico, por um período de 120 dias.

Os fungos de armazenagem comumente desenvolvem-se em grãos de milho com menos de $18 \%$ de umidade, e os mais importantes pertencem aos gêneros Aspergillus e Penicillium. Espécies de Aspergillus podem desenvolver-se em grãos com umidade tão baixa como 13,1\% e as espécies de Penicillium desenvolvem-se ativamente acima de 16\% (Pinto, 1996).

$\mathrm{Na}$ espera pela secagem de grãos de mi1ho, os tratamentos desses grãos com thiabendazole, ácido acético, uréia ou propionato de cálcio + aluminiossilicatos são eficientes no controle do mofo causado por A. flavus (Pinto, 2004). A aeração contínua e a proteção dos grãos com os fungicidas benomyl, thiabendazole e fludioxonil também são procedimentos eficientes para reduzir tanto o mofo incitado pelos fungos A. flavus e Penicillium spp., como a biossíntese de aflatoxinas (Pinto et al., 2005).

Fungos do gênero Aspergillus, principalmente A.flavus e A. parasiticus, são produtores das aflatoxinas, micotoxinas altamente tóxicas ao ser humano e aos animais domésticos, que podem ocorrer tanto antes da colheita como no armazenamento. Por outro lado, diferenças significativas de infecção de grãos por A. flavus, em diferentes híbridos de milho, são relatadas por McMillian et al. (1980), Windstrom et al. (1981), King \& Scott (1981, 1982) e Zummo \& Scott (1985).

O objetivo deste trabalho foi comparar, em grãos colhidos úmidos e armazenados em sis- 
temas com e sem aeração, cultivares de milho quanto à produção de grãos com mofo por $A$. flavus e Penicillium spp.

Compararam-se as cultivares BR 206, BRS 2114, BRS 2110, BRS 1001, BRS 2020, BRS 3151, BR 3123, BRS 1010, BRS 3060, BRS 3003, CMS 200.122 e BRS 1030. Imediatamente após a colheita e a pré-limpeza, os grãos (100 $\mathrm{kg}$ por parcela) foram armazenados em camada de 51,8 cm de espessura, por nove dias, em sistema com aeração - SCA (tambores metálicos de 200 L de capacidade, com fundos seccionados e substituídos por tela fina de aço, colocados sobre dutos de aeração), e em sistema sem aeração - SSA (tambores com fundo de metal, sobre estrados de madeira). Os tambores tinham dimensões de $0,82 \mathrm{~m}$ de altura e $0,60 \mathrm{~m}$ de diâmetro. $\mathrm{O}$ sistema com aeração foi dotado de quatro dutos com formato trapezoidal $(6,0 \times 1,1 \times 0,7 \mathrm{~m})$, possuindo cada duto nove orifícios de aeração $(0,54$ $\mathrm{m}$ de diâmetro), sobre os quais foram colocados os tambores com a massa de grãos de milho. A aeração contínua com ar ambiente foi promovida por um ventilador de $2750 \mathrm{rpm}$, motor de 0,5 HP, vazão de ar de 0,42 m³/seg (Pinto, 2001). Para cada cultivar, $300 \mathrm{~kg}$ de grãos (três repetições de $100 \mathrm{~kg}$ ) foram colocados em sistema com aeração e $300 \mathrm{~kg}$ em sistema sem aeração. O delineamento experimental foi o inteiramente casualizado, com 24 tratamentos em três repetições.

No Laboratório de Patologia de Sementes e Grãos, da Embrapa Milho e Sorgo, Sete Lagoas - MG, quantificou-se, visualmente aos dois, seis e nove dias de armazenagem, a percentagem de grãos com mofo por A. flavus (colônias fúngicas verde-oliva a verde-amarelado) e Penicillium spp. (colônias fúngicas azul claro, cinza ou verde claro). Nessas mesmas épocas, procedeu-se ao monitoramento dos teores de umidades dos grãos das parcelas experimentais, utilizando-se o método de estufa com circulação natural de ar, regulada em $105 \pm 3{ }^{\circ} \mathrm{C}$, durante 24 horas, de acordo com as Regras de Análise de Sementes (Brasil, 1992). Amostras individuais, de aproximadamente $300 \mathrm{~g}$ de grãos, foram retiradas nas 72 parcelas experimentais, para a quantificação de grãos com mofo e para o monitoramento dos teores de umidade. A temperatura intergranular, nas parcelas experimentais, foi monitorada diariamente, usando-se geotermômetros.

As análises de variância (DIC) e a comparação visual das médias dos grãos com mofo (teste de Tukey 5\%), nos dois sistemas e períodos de armazenagem, foram realizadas empregando-se o programa MSTAT-C, versão 1.3, da Michigan State University.

A determinação visual da percentagem de grãos com mofo, na cultivar BR 206, foi semelhante à das cultivares BRS 2114 e BRS 2110, com baixas incidências de mofo em seus grãos, nos sistemas com e sem aeração, durante o período de nove dias, de armazenagem (TABELA 1). O percentual de mofo nas cultivares BRS 1001, BRS 2020, BRS 3151 e BR 3123 foi semelhante até 6 dias de armazenagem, porém, aos 9 dias no sistema com aeração ocorreu maior percentual em BRS 3151. Contudo, no sistema sem aeração, a cultivar BRS 3151, com maior percentagem de mofo, diferiu apenas da cultivar BR 3123. Nos períodos de seis e nove dias de armazenagem, houve menor incidência de mofo nos grãos de BRS 3060 que nas cultivares BRS 1010, BRS 3003, CMS 200.122 e BRS 1030, no sistema sem aeração. Resultado semelhante foi obtido aos nove dias em armazenagem com aeração. Na cultivar BRS 1030 houve maior percentual de grãos com mofo aos nove dias de armazenagem com aeração que nas demais cultivares, o que poderia estar associado ao elevado 
percentual de umidade dos grãos na colheita. Entretanto, a cultivar BRS 3060 apresentou-se com menores percentuais de grãos com mofo entre as cultivares, nas três épocas de avaliação, em ambos os sistemas de armazenagem, apesar de ter sido colhida com maior umidade. Assim, sugere-se que outros fatores podem influir no percentual de mofo em grãos, além de sua umidade no momento da colheita. No sistema com aeração, até o $2^{\circ}$ dia de armazenagem, não houve diferença significativa entre as cultivares com relação ao mofo de seus grãos. Contudo, a partir do $6^{\circ}$ dia de armazenagem, ocorreram diferenças significativas entre as cultivares avaliadas. No $9^{\circ}$ dia de armazenagem, os grãos das cultivares BR 3123 e BRS 3060, colhidos com 19,8 e $21,3 \%$ de umidade, respec- tivamente, tiveram baixo incidência de grãos com mofo, à semelhança dos grãos da cultivar BR 206, colhidos com 17,2\% de umidade. Tal fato corrobora a afirmativa de que, no processo de colonização de grãos de milho por fungos de armazenagem (A. flavus e Penicillium spp.), além do binômio temperatura e umidade, há de se considerar a interação fungo e genótipo, isto é, temperatura intergranular, a umidade dos grãos e cultivar utilizada. As cultivares BRS 1010 e BRS 3060, colhidas com a mesma umidade, diferiram significativamente entre si, com relação à produção de grãos visualmente com mofo, aos dois, seis e nove dias de armazenagem, no sistema não aerado, e os seis e nove dias, no sistema aerado. Adicionalmente, aos 9 dias de armazenagem e em

TABELA 1. Percentagem de grãos de milho visualmente com mofo, armazenados imediatamente após a colheita, de diferentes cultivares, em sistemas com e sem aeração. Embrapa Milho e Sorgo, Sete Lagoas, MG, 2003.

\begin{tabular}{|c|c|c|c|c|c|c|c|c|}
\hline \multirow{3}{*}{ Cultivar } & \multirow{3}{*}{$\begin{array}{c}\text { Umidade grãos } \\
\text { na colheita } \\
(\%)\end{array}$} & \multirow{3}{*}{$\begin{array}{c}\text { Grãos com } \\
\text { mofo na } \\
\text { colheita }(\%)^{1}\end{array}$} & \multicolumn{6}{|c|}{ Grãos com mofo durante armazenagem $(\%)^{2}$} \\
\hline & & & $\mathrm{SCA}^{3}$ & SSA & SCA & SSA & SCA & SSA \\
\hline & & & \multicolumn{2}{|c|}{2 dias de armazenagem } & \multicolumn{2}{|c|}{6 dias de armazenagem } & \multicolumn{2}{|c|}{9 dias de armazenagem } \\
\hline BR 206 & 17,2 & 0,39 & $0,20 \mathrm{a}$ & $0,05 \mathrm{bc}$ & $0,17 \mathrm{e}$ & 4,14 cde & $0,26 \mathrm{~g}$ & $3,37 \mathrm{fg}$ \\
\hline BRS 2114 & 18,3 & 0,21 & $0,00 \mathrm{a}$ & $0,00 \mathrm{c}$ & $0,97 \mathrm{e}$ & $0,77 \mathrm{e}$ & $0,29 \mathrm{~g}$ & $0,67 \mathrm{~g}$ \\
\hline BRS 2110 & 18,5 & 0,00 & $0,41 \mathrm{a}$ & $0,26 \mathrm{bc}$ & $0,79 \mathrm{e}$ & 3,24 de & $0,87 \mathrm{~g}$ & 5,36 defg \\
\hline BRS 1001 & 19,1 & 0,53 & $0,75 \mathrm{a}$ & $0,82 \mathrm{abc}$ & $1,52 \mathrm{e}$ & $8,39 \mathrm{bc}$ & $1,18 \mathrm{fg}$ & 8,71 cde \\
\hline BRS 2020 & 19,2 & 0,13 & $0,46 \mathrm{a}$ & $0,75 \mathrm{abc}$ & $1,64 \mathrm{e}$ & $5,96 \mathrm{~cd}$ & $1,16 \mathrm{~g}$ & $7,30 \mathrm{def}$ \\
\hline BRS 3151 & 19,5 & 0,17 & $0,32 \mathrm{a}$ & $0,32 \mathrm{bc}$ & $1,88 \mathrm{de}$ & $7,55 \mathrm{bcd}$ & 3,06 de & $9,41 \mathrm{~cd}$ \\
\hline BR 3123 & 19,5 & 0,22 & $0,03 \mathrm{a}$ & $0,42 \mathrm{bc}$ & $1,74 \mathrm{de}$ & 3,98 cde & $1,27 \mathrm{fg}$ & 4,53 efg \\
\hline BRS 1010 & 21,2 & 0,46 & $0,50 \mathrm{a}$ & $2,02 \mathrm{a}$ & $5,59 \mathrm{bc}$ & $12,27 \mathrm{ab}$ & $4,58 \mathrm{c}$ & $14,67 \mathrm{ab}$ \\
\hline BRS 3060 & 21,3 & 0,17 & $0,49 \mathrm{a}$ & $0,43 \mathrm{bc}$ & $1,90 \mathrm{de}$ & $5,05 \mathrm{cde}$ & $2,23 \mathrm{fg}$ & $7,41 \mathrm{def}$ \\
\hline BRS 3003 & 21,4 & 0,29 & $2,41 \mathrm{a}$ & $1,36 \mathrm{ab}$ & $4,44 \mathrm{~cd}$ & $15,99 \mathrm{a}$ & $6,98 \mathrm{~b}$ & $17,83 \mathrm{a}$ \\
\hline CMS 200.122 & 21,9 & 0,12 & $1,18 \mathrm{a}$ & $0,78 \mathrm{abc}$ & $7,48 \mathrm{ab}$ & $11,20 \mathrm{ab}$ & $3,28 \mathrm{~d}$ & $15,64 \mathrm{ab}$ \\
\hline BRS 1030 & 22,9 & 0,35 & $0,93 \mathrm{a}$ & $1,18 \mathrm{abc}$ & $9,63 \mathrm{a}$ & $13,84 \mathrm{a}$ & $9,55 \mathrm{a}$ & $12,36 \mathrm{bc}$ \\
\hline C.V $(\%)$ & & & 40,48 & 48,9 & 30,22 & 21,70 & 22,33 & 30,82 \\
\hline
\end{tabular}

${ }^{1}$ Penicillium sp., ${ }^{2}$ Aspergillus flavus e Penicillium spp. (predominância de A. flavus), ${ }^{3}$ Sistema com Aeração (SCA) e Sistema sem Aeração (SSA), ${ }^{4}$ Médias seguidas pelas mesmas letras, nas colunas, não diferem entre si pelo teste de Tukey $(\mathrm{P}<5 \%)$. 
ambos sistemas, as cultivares BRS 3151 e BRS 3123 tiveram comportamento diferencial. Esses resultados comprovam que há interação genótipo-patógeno (cultivar - A. flavus/ Penicillium spp.) e corroboram aqueles apresentados McMillian et al. (1980), Windstrom et al. (1981), King \& Scott $(1981,1982)$ e Zummo \& Scott (1985).

Com relação aos monitoramentos da temperatura intergranular e da umidade dos grãos (Tabela 2), verifica-se que, no sistema com aeração, a temperatura intergranular média manteve-se adequada durante o período da armazenagem dos grãos $\left(21,2\right.$ a $\left.22,8^{\circ} \mathrm{C}\right)$, enquanto que, no sistema sem aeração, além de mais elevadas, as temperaturas apresentaram também maior amplitude de variação $\left(27,9\right.$ a $\left.37,1^{\circ} \mathrm{C}\right)$. Ademais, durante o período de armazenagem, a cul- tivar BR 3123, colhida com 19,8\%, teve comportamento semelhante ao das cultivares BR 206, BRS 2114 e BRS 2110, colhidas com 17,2; 18,3 e $18,5 \%$ de umidade, respectivamente. Isso sugere que alguns genótipos podem ser colhidos com umidade mais elevada, sem reflexos deletérios na sanidade de seus grãos, permitindo uma adequada armazenabilidade dos mesmos. Esses resultados são diferentes dos obtidos por Alves et al. (2001), que avaliaram os danos em grãos da cultivar AG 1051, em quatro teores de umidade de colheita, mas sendo secados até a umidade final de $13 \%$ b.u. e, só em seguida, armazenados.

Do exposto, pode-se concluir que há, na armazenagem, diferenças entre genótipos de milho com relação à produção de grãos com mofo causado por A. flavus e Penicillium spp.

TABELA 2. Monitoramento da temperatura intergranular e da umidade em grãos de milho armazenados, imediatamente após a colheita, em sistemas com e sem aeração. Embrapa Milho e Sorgo, Sete Lagoas, MG, 2003.

\begin{tabular}{|c|c|c|c|c|c|c|c|c|}
\hline \multirow{3}{*}{ Cultivar } & \multirow{2}{*}{\multicolumn{2}{|c|}{$\begin{array}{c}\text { Temperatura } \\
\text { intergranular }\left({ }^{\circ} \mathrm{C}\right)^{1}\end{array}$}} & \multicolumn{6}{|c|}{ Umidade dos grãos durante armazenagem (\%) } \\
\hline & & & $\mathbf{S C A}^{2}$ & SSA $^{2}$ & SCA & SSA & SCA & SSA \\
\hline & SCA & SSA & \multicolumn{2}{|c|}{2 dias de armazenagem } & \multicolumn{2}{|c|}{6 dias de armazenagem } & \multicolumn{2}{|c|}{9 dias de armazenagem } \\
\hline BR 206 & 22,3 & 28,6 & 15,4 & 16,1 & 15,3 & 15,5 & 14,2 & 16,1 \\
\hline BRS 2114 & 21,7 & 27,9 & 16,3 & 16,3 & 15,6 & 15,3 & 14,3 & 15,0 \\
\hline BRS 2110 & 23,2 & 29,0 & 15,9 & 16,3 & 15,2 & 15,7 & 15,0 & 15,3 \\
\hline BRS 1001 & 22,5 & 33,3 & 17,8 & 18,4 & 16,4 & 17,0 & 15,4 & 15,2 \\
\hline BRS 2020 & 22,7 & 31,6 & 17,5 & 18,0 & 17,2 & 14,2 & 15,3 & 15,7 \\
\hline BRS 3151 & 22,8 & 33,3 & 18,1 & 17,8 & 18,0 & 15,1 & 16,6 & 16,3 \\
\hline BR 3123 & 21,2 & 32,1 & 18,1 & 18,3 & 17,3 & 17,4 & 17,5 & 15,4 \\
\hline BRS 1010 & 22,3 & 37,1 & 18,3 & 20,7 & 17,5 & 18,4 & 16,7 & 18,2 \\
\hline BRS 3060 & 22,8 & 33,2 & 18,9 & 19,9 & 17,6 & 17,9 & 18,1 & 17,9 \\
\hline BRS 3003 & 22,3 & 34,3 & 19,5 & 19,9 & 19,5 & 16,7 & 15,9 & 17,4 \\
\hline CMS 200.122 & 21,9 & 32,6 & 19,0 & 20,9 & 18,1 & 18,3 & 19,1 & 17,7 \\
\hline BRS 1030 & 22,7 & 35,8 & 20,6 & 21,5 & 19,1 & 18,4 & 18,7 & 19,4 \\
\hline
\end{tabular}




\section{Agradecimentos}

A Osni Alves da Silva, José Moreira Campos e Ademar Verneque (Assistentes de Operações da Embrapa Milho e Sorgo) e a Gilberto Ribeiro Rodrigues (Operário Rural) a valiosa colaboração na condução deste trabalho.

\section{Literatura citada}

ALVES, W. M.; FARONI, L. R. D.; CORRÊA, P. C.; QUEIROZ, D. M.; TEIXEIRA, M. M. Influência dos teores de umidade de colheita na qualidade do milho (Zea mays L.) durante o armazenamento. Revista Brasileira de Armazenagem, v.26, n.2, p.40-45, 2001.

BRASIL. Ministério da Agricultura e Reforma Agrária. Regras para Análise de Sementes. Brasília, DF. 1992. 365p.

KING, S. B.; SCOTT, G. E. Screening maize for resistance to kernel infection by Aspergillus flavus. Phytopathology, v.71, p.231 (Abstract), 1981.

KING, S. B.; SCOTT, G. E. Field inoculation techniques to evaluate maize for reaction to kernel infection by Aspergillus flavus. Phytopathology, v.72, p.782-785, 1982.

MCMILLIAN, W. W.; WINDSTROM, N. W.; WILSON, D. M.; HILL, R. A. Transmission by maize weevils of Aspergillus flavus and its survival on selected corn hybrids. Journal
Economic Entomology, v.73, p.793-794, 1980.

PINTO, N. F. J. A. Controle de patógenos em grãos de milho armazenados. Summa Phytopathologica, v.22, n.1, p.77-78, 1996.

PINTO, N. F. J. A. Tratamento químico de grãos de sorgo úmidos visando o controle de fungos de armazenamento. Revista Brasileira de Armazenagem, v.26, n.2, p.55-59, 2001.

PINTO, N. F. J. A. Preservação da qualidade sanitária de grãos úmidos de milho através do controle da atividade fúngica. Revista Brasileira de Armazenagem, v.29, n.2, p.159-164, 2004.

PINTO, N. F. J. A.; PRADO, G.; OLIVEIRA, M. S. Proteção química e biológica de grãos de milho úmidos contra fungos de armazenagem e produção de aflatoxinas. Revista Brasileira de Milho e Sorgo, v.4, n.2, p.172-179, 2005.

WINDSTROM, N. W.; WILSON, D. M.; MCMILLIAN, W. W. Aflatoxin contamination of preharvest corn as influenced by timing and method of inoculation. Applied Environmental Microbiology, v.42, p.249-251, 1981.

ZUMMO, N.; SCOTT, G. E. Evaluation of field inoculation techniques for screening corn genotypes against kernel infection by Aspergillus flavus in Mississippi. Phytopathology, v.75, p.1330, 1985. 